\title{
A GPS-Based Framework for Understanding Outdoor Mobility Patterns of Older Adults with Dementia: An Exploratory Study
}

\author{
Sayeh Bayat ${ }^{a, b} \quad$ Gary Naglie $^{b, c, d, e, f} \quad$ Mark J. Rapoport ${ }^{g, h} \quad$ Elaine Stasiulis ${ }^{d, i}$ \\ Michael J. Widener ${ }^{j} \quad$ Alex Mihailidis $^{a}, b, k$ \\ anstitute of Biomedical Engineering, University of Toronto, Toronto, ON, Canada; ${ }^{b} \mathrm{KITE}$ Research Institute, Toronto \\ Rehabilitation Institute, Toronto, ON, Canada; 'Department of Medicine, Baycrest Health Sciences, Toronto, ON,

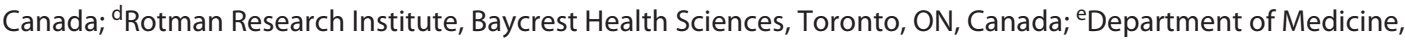

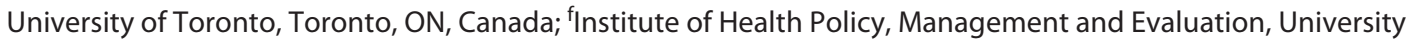 \\ of Toronto, Toronto, ON, Canada; 9 Department of Psychiatry, Sunnybrook Health Sciences Centre, Toronto, ON, \\ Canada; ' hepartment of Psychiatry, University of Toronto, Toronto, ON, Canada; 'Institute of Medical Science, \\ University of Toronto, Toronto, ON, Canada; 'Department of Geography and Planning, University of Toronto, \\ Toronto, ON, Canada; 'Department of Occupational Therapy \& Occupational Science, University of Toronto, \\ Toronto, ON, Canada
}

\section{Keywords}

Older adults · Dementia - Global positioning system ·

Outdoor mobility · Life space

\begin{abstract}
Introduction: An active lifestyle may protect older adults from cognitive decline. Yet, due to the complex nature of outdoor environments, many people living with dementia experience decreased access to outdoor activities. In this context, conceptualizing and measuring outdoor mobility is of great significance. Using the global positioning system (GPS) provides an avenue for capturing the multi-dimensional nature of outdoor mobility. The objective of this study is to develop a comprehensive framework for comparing outdoor mobility patterns of cognitively intact older adults and older adults with dementia using passively collected GPS data. Methods: A total of 7 people with dementia (PwD) and 8 cognitively intact controls (CTLs), aged 65 years or old-
\end{abstract}

er, carried a GPS device when travelling outside their homes for 4 weeks. We applied a framework incorporating 12 GPSbased indicators to capture spatial, temporal, and semantic dimensions of outdoor mobility. Results: Despite a small sample size, the application of our mobility framework identified several significant differences between the 2 groups. We found that PwD participated in more medical-related (Cliff's Delta $=0.71,95 \%$ Cl: $0.34-1$ ) and fewer sport-related (Cliff's Delta $=-0.78,95 \% \mathrm{Cl}:-1$ to -0.32 ) activities compared to the cognitively intact CTLs. Our results also suggested that longer duration of daily walking time (Cliff's Delta $=0.71$, 95\% Cl: $0.148-1)$ and longer outdoor activities at night, after 8 p.m. (Hedges' $\mathrm{g}=1.42,95 \% \mathrm{Cl}$ : $0.85-1.09$ ), are associated with cognitively intact individuals. Conclusion: Based on the proposed framework incorporating 12 GPS-based indicators, we were able to identify several differences in outdoor mobility in PwD compared with cognitively intact CTLs.

(c) 2021 The Author(s)

Published by S. Karger AG, Basel
C 2021 The Author(s)

Published by S. Karger AG, Basel

This is an Open Access article licensed under the Creative Common Attribution-NonCommercial-4.0 International License (CC BY-NC) (http://www.karger.com/Services/OpenAccessLicense), applicable to the online version of the article only. Usage and distribution for commercial purposes requires written permission.
Correspondence to:

Sayeh Bayat, sayeh.bayat@mail.utoronto.ca 


\section{Introduction}

Outdoor mobility is fundamental for healthy ageing. In fact, preserving mobility contributes to various health benefits including reduced risk of depression $[1,2]$, improved functional ability including instrumental activities of daily living [3], and preserved cognitive functions for older adults $[4,5]$. Unfortunately, maintaining a mobile lifestyle becomes more challenging with advancing age. A major threat to maintaining outdoor mobility is dementia [6], particularly Alzheimer's type, because it impairs cognitive and functional abilities that affect complex activities such as spatial navigation and driving $[7,8]$. As a result of navigational impairment, people with dementia $(\mathrm{PwD})$ may become lost in familiar or unfamiliar environments [9]. Thus, despite the benefits of outdoor environments, PwD frequently experience loss of confidence or anxiety in relation to going outdoors [10]. These effects may lead them to gradually move within smaller areas as their cognitive abilities decline, or may even stop them from going outside altogether [10]. Furthermore, older adults who have been diagnosed with dementia may eventually stop driving for their personal and the public's safety [11]. Given that driving is the most common form of transportation for older adults [12], the loss of driving privileges makes it even more challenging for PwD to maintain a mobile lifestyle. For instance, in the USA, older non-drivers are on average 65\% less likely to participate in social, family, and religious activities, $59 \%$ less likely to go shopping or visit a restaurant, and 15\% less likely to visit a doctor compared to older drivers [13]. Considering these challenges and the impact of reduced outdoor mobility on the daily lives of $\mathrm{PwD}$, there is an increased need for conceptualizing and measuring mobility.

A commonly used mobility measure for older adults is life space, defined as the spatial area through which an individual moves within a specific period [14]. Life space is an important indicator of older adults' physical and cognitive health, as well as mental wellbeing, because it provides some insight into a person's level of engagement with the environment. Previous studies have demonstrated associations between life space and observed physical performance and self-reported function [15]. Reduced life space mobility is also interrelated with a higher prevalence of depressive symptoms [16], lower health-related quality of life [17], and increased fear of falling [18]. Furthermore, previous studies have reported that life space is a significant predictor of cognitive decline, and a larger life space is associated with reduced cognitive decline in older adults [19]. Considering that a larger life space, which generally indicates that an older adult is more ac-

Mobility Patterns of Older Adults with Dementia tive and engaged, may protect against cognitive decline in older adults, it is important to accurately and objectively examine the relationship between environmental movements and cognitive function.

\section{Traditional Life Space Assessments}

May et al. [20] introduced the first measurement of life space, the life space diary. In this method, life space is divided into 5 concentric zones: (1) the bedroom, (2) the rest of the house, (3) the garden or yard, or the pavement surrounding the house, (4) the "block" where the house is located at, and (5) the area across a traffic-bearing street. This method requires the participants to keep records of the zones in which they moved during the day, every day for 1 month.

To reduce the burden of recording the movements every day, the Life Space Assessment Questionnaire (LSAQ) was introduced [14]. LSA-Q consists of 9 questions and asks participants whether they have been to certain zones within their environment in the past 3 days and captures a broader range of environmental regions compared to the life space diary. However, the time period of 3 days in LSA-Q may underrepresent the mobility of older adults, and since it again relies on self-report, it may be influenced by recall bias [21].

Later, the University of Alabama at Birmingham LifeSpace Assessment (UAB-LSA) was introduced, which was a more detailed life space measure [22]. This tool evaluates mobility of participants during the 4 weeks before the assessment and provides a single number ranging from 0 to 120 to reflect the lifestyle of a person (a higher score shows a more active lifestyle) and their frequency of movement as well as the level of assistance they require for movement. UAB-LSA involves a single interview requiring the individuals to recall their past daily activities; thus, it may also be influenced by recall bias.

These self-reported life space measures are especially limited among the cognitively impaired population. Individuals with dementia may not be able to reliably recall their prior movements due to memory impairment [23], and proxy assessments completed by care partners may be prone to bias [24]. In addition, these measures are usually completed only a few times with each individual; therefore, it is not possible to continuously monitor the changes in life space and relate them to the progress of dementia or changes in cognitive functioning over time.

\section{GPS-Based Life Space Measures}

To eliminate the various limitations of the self-report measures, mobility monitoring systems that use the glob- 
al positioning system (GPS) to track locations are recommended as a tool to record movement in the environment [25]. In a proof-of-principal study [26], the life space of 3 adults aged 48 years or younger was measured using a combination of GPS sensors and in-home Bluetooth transmitters and the scoring scheme from UAB-LSA. Later, another study proposed using the area of an ellipse covering all the GPS coordinates tracked, total distance travelled, and the maximum distance travelled from home as qualitative measures for life space to compare life space of an older adult and a university student for 5 consecutive days [27]. Both of these initial studies were conducted on a small sample size $(n<4)$ of healthy individuals. In the context of cognitively impaired populations, to the best of our knowledge, only 1 study has assessed the utility of a GPS-based life space measure. This study used a small set of GPS-based indicators including area and perimeter of the smallest polygon containing all the tracked GPS coordinates, percentage of time away from home, and average travelled distance to provide quantitative measures for life space of older adults with mild-tomoderate Alzheimer's disease over a relatively short 3- to 5-day period [24].

These previous studies only focus on quantifying life space using passively collected GPS data. It is unlikely that life space alone captures the entire complexity of living in the environment. Rather, life space measures only capture the extent and, in some cases, the frequency of excursions into the outdoor environment. To provide a better understanding of different dimensions of outdoor mobility in health and ageing research, a few studies have attempted to categorize and classify mobility indicators [28-31]. Of note is the study conducted by Fillekes et al. [31] in which a framework for classification of GPS-based mobility indicators is introduced. This framework categorizes indicators based on their characteristic aspects (i.e., indicators' content relevant to space, time, and movement scope), as well as analytical aspects (i.e., how a mobility indicator is summarized with respect to temporal scale and statistics). Although this framework complements existing life space studies that underrepresent temporal characteristics of mobility, it does not capture more complex out-of-home behaviours of older adults (e.g., activity types). Capturing these higher complexity behaviours is particularly important for PwD because they can be indicative of the cognitive status of a person [32]. Furthermore, Fillekes et al.'s [31] framework was designed for healthy older adults. However, in the context of the cognitively impaired population, it may be the case that different dimensions of outdoor mobility display dif- ferent strengths of relationship with cognitive status. Furthermore, in another framework proposed by Wettstein et al. [32], the outdoor mobility behaviours of community-dwelling older adults with and without cognitive impairment were investigated. Although this work extends the available literature on the life space of PwD to include walking behaviour and high complexity activities, it relies on both GPS devices and questionnaires to collect mobility data and explore outdoor behaviours. Thus, there are no studies, to date, that rely solely on GPS technology to provide a holistic overview of outdoor mobility of cognitively impaired populations. In light of the limitations of the previous studies, the goal of the present study is to further investigate the application of GPS technology to develop and apply a framework for comparing outdoor mobility patterns of cognitively intact older adults and older adults with dementia based on a comprehensive set of indicators reflecting different dimensions of outdoor mobility.

\section{Materials and Methods}

The feasibility of using GPS technology to quantify outdoor mobility patterns was evaluated by comparing the everyday behaviour of a group of individuals with dementia $(\mathrm{PwD})$ to a group of cognitively intact older adults (CTL).

\section{Data Collection}

Participants

Participants included 7 PwD diagnosed by a specialist and who lived with a family care partner (e.g., spouse or family member) and 8 cognitively intact community-dwelling older adult controls (CTLs) [33]. The participants were recruited through the Baycrest Health Sciences database of research volunteers as well as printed and electronic advertisements on flyers, magazines, social media sites, and the University of Toronto alumni email list. Both groups were drawn from the Greater Toronto Area, predominantly urban areas with similar infrastructure (i.e., access to public transit and roads) and weather. Candidates for inclusion in the CTL group were screened for cognitive impairment using the Montreal Cognitive Assessment (MoCA). The recommended cutoff score for normal cognition of 26 was adopted [34]. All participants were 65 years or older, lived in their own homes, and were independent in their functioning (assessed using Older Americans Resources and Services [OARS] ADL Scale [35]). Informed consent was obtained from all participants. Ethical approval was obtained from the Baycrest Health Sciences Ethics Committee and the University Health Network Ethics Committee.

Potential CTL participants were screened for cognitive impairment over the telephone using the self-rated memory complaint questionnaire (MAC-Q) [36]. Individuals who fulfilled the initial criteria were then invited for an in-person meeting to obtain written consent and administer assessment tools including the MoCA. Inclusion and exclusion criteria for the study are shown in Table 1 . 
Table 1. Inclusion and exclusion criteria for the dementia group and the control group

\begin{tabular}{|c|c|}
\hline Inclusion criteria & Inclusion criteria \\
\hline Be aged 65 years or older & Be aged 65 years or older \\
\hline Psychiatrist, Neurologist, or Geriatrician) & Live independently in their own home (assessed using ADL scale) \\
\hline Have a live-in caregiver & Have an absence of morbidity that significantly impacts mobility \\
\hline Live in their own homes & (assessed using Charlson Comorbidity assessment) \\
\hline Exclusion criteria & Exclusion criteria \\
\hline Wear a pacemaker & Wear a pacemaker \\
\hline
\end{tabular}

Table 2. Proposed set of GPS-based mobility indicators

\begin{tabular}{|c|c|c|c|c|c|c|c|c|c|c|}
\hline & name & abbreviation & count & extent & duration & timing & Semantic & stop & move & $\begin{array}{l}\text { Temporal } \\
\text { scale }\end{array}$ \\
\hline 2 & Radius of gyration & RG & & $\checkmark$ & & & & $\checkmark$ & & Global \\
\hline 3 & Life space area & LSArea & & $\checkmark$ & & & & $\checkmark$ & & Daily \\
\hline 4 & Destinations, $n$ & nDest & $\checkmark$ & & & & & $\checkmark$ & & Global \\
\hline 7 & Time doing out-of-home activities & TOA & & & $\checkmark$ & & & & $\checkmark$ & Daily \\
\hline 8 & Time on foot & ToF & & & $\checkmark$ & & $\checkmark$ & & $\checkmark$ & Daily \\
\hline 9 & Time in vehicle & $\mathrm{TiV}$ & & & $\checkmark$ & & $\checkmark$ & & $\checkmark$ & Daily \\
\hline 10 & Trip time period & TTP & & & & $\checkmark$ & & & $\checkmark$ & Daily \\
\hline 11 & Outdoor activity duration time period & OADurTP & & & & $\checkmark$ & & $\checkmark$ & & Daily \\
\hline 12 & Types of activities & ActivityType & & & & & $\checkmark$ & $\checkmark$ & & Global \\
\hline
\end{tabular}

Global indicators are assessed over the entire period of the study (4 weeks). GPS, global positioning system.

GPS Data Collection

Upon enrolment in the study, participants were introduced to the SafeTracks Prime Mobile GPS device (SafeTracks GPS Canada, Red Deer, AB, CA) and instructed on how to use and charge it. Participants were asked to carry these devices with them during every excursion outside their home. Care partners of the participants with dementia were also provided with instructions about use and maintenance of the device so that they could assist their partners throughout the study. To increase compliance, the participants received regular reminders in the form of emails and phone calls. Participants in both groups had at most 1 day of missed data during the 4 -week period.

In addition, participants were provided with a small booklet and were instructed to record details describing their daily mobility patterns, including time and destination information. For individuals with dementia, their care partners were asked to complete the travel diary recording. All participants completed 4 weeks of GPS data collection and travel diary recording. The 4-week study period was selected based on the time period used in the traditional life space assessments including UAB-LSA [20, 22].

Mobility Patterns of Older Adults with Dementia

\section{Measures}

A questionnaire was administered to collect information about demographics, living arrangements, driving history, and current transportation patterns of the participants. In addition, the UABLSA was administered at baseline and the end of data collection to provide an additional measure of the extent of participants' community mobility. Participant's physical and instrumental activities of daily living were assessed using the OARS ADL scale [35]. Participants were also assessed for comorbid conditions using the Charlson Comorbidity Index (CCI) [37]. Finally, participants' neighbourhood walkability was assessed using the "Walk Score" measure [38].

\section{GPS-Based Outdoor Mobility Classification Framework}

The framework presented is an extension of the classification framework described by Fillekes et al. [31]. First, each individual's overall trajectory was segmented into stops (i.e., destinations) and moves (i.e., trips between destinations). The trajectory segmentation method is described by Bayat et al. [39]. In this approach, the periods during which a participant did not move for at least $3 \mathrm{~min}$ 
Table 3. Description of the GPS-based mobility indicators

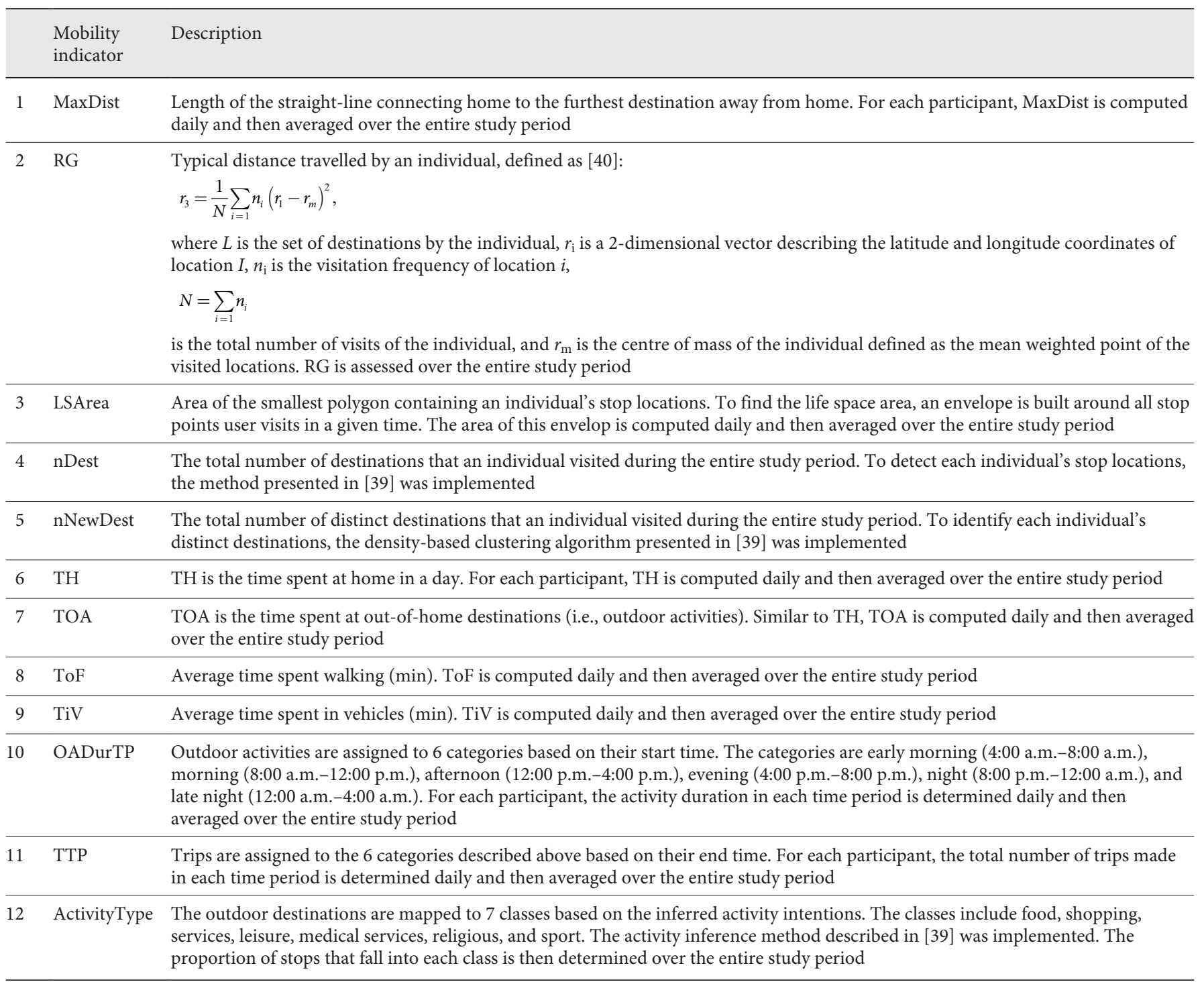

GPS, global positioning system; MaxDist, maximum distance from home; RG, radius of gyration; LSArea, life space area; nDest, number of destinations; nNewDest, number of new destinations; TH, time at home; TOA, time doing out-of-home activities; ToF, time on foot; TiV, time in vehicle; TTP, trip time period; OADurTP, outdoor activity duration time period; ActivityType, types of activities.

were detected and labelled as "destinations," and periods of movement with a distance of $>150 \mathrm{~m}$ were identified and extracted as "trips." If a participant's GPS signal was unavailable, the last known location of the user was compared against a list of subway station locations to identify potential underground transit trips. Then, similar to [31], we identified GPS-based mobility indicators that describe the spatial distribution of GPS data or temporal dimensions of mobility. Spatial indicators either refer to the number of mobility-related events (i.e., count) or the spatial size of mobilityrelated events (i.e., extent) [31]. Temporal indicators, on the other hand, either reflect the time spent at a mobility-related event (i.e., duration) or the time of occurrence of a mobility-related event (i.e., timing). We also added indicators that describe the semantic di- mensions of outdoor mobility. These indicators enable an exploration of the reasons behind the patterns that emerge in space and time (i.e., motivations and intentions). For example, using semantic dimensions, we can explain that the motivation behind a trip to a particular destination (i.e., activity type) was to visit a doctor. To infer activity types, we used the activity inference approach described by Bayat et al. [39]. In this approach, a distance-based probabilistic model is utilized based on Google Places Application Programming Interface [31] to retrieve information about a particular place. Using this information, places were mapped to 7 categories: food, shopping, services, leisure, medical services, religious, and sport. Finally, all GPS-based indicators are used to describe stops or moves and are either assessed over the entire study 
Table 4. Participants' demographics and assessed characteristics

\begin{tabular}{|c|c|c|c|c|}
\hline & $\begin{array}{l}\text { CTL group } \\
(n=8)\end{array}$ & $\begin{array}{l}\text { PwD group } \\
(n=7)\end{array}$ & $p$ value & $t$ \\
\hline Age, years & $72.9 \pm 5.9$ & $79.3 \pm 6.3$ & 0.06 & -2.05 \\
\hline $\mathrm{MoCA}^{\mathrm{a}}$ & $27.5 \pm 1.2$ & $19.4 \pm 2.4$ & $0.004^{*}$ & 4.68 \\
\hline Education, years & $16.5 \pm 3.6$ & $14.1 \pm 3.4$ & 0.16 & 1.49 \\
\hline Female gender, $n$ & 4 & 3 & & \\
\hline CCI & $1.13 \pm 1.4$ & $2.14 \pm 1.6$ & 0.21 & -1.33 \\
\hline UAB-LSA $(1)^{\mathrm{b}}$ & $75.5 \pm 13.2$ & $54.9 \pm 28.7$ & 0.12 & 1.75 \\
\hline UAB-LSA $(2)^{\mathrm{b}}$ & $74.0 \pm 12.3$ & $52.5 \pm 21.0$ & 0.06 & 2.16 \\
\hline Physical ADL & $13.8 \pm 0.7$ & $11.9 \pm 2.5$ & 0.09 & 1.95 \\
\hline Instrumental ADL & $14.0 \pm 0.0$ & $8.9 \pm 5.1$ & $0.04^{*}$ & 2.66 \\
\hline Walk score of Home & $73.3 \pm 22.3$ & $61.0 \pm 33.5$ & 0.4 & 0.82 \\
\hline
\end{tabular}

${ }^{*} p<0.05 .{ }^{\text {a }}$ Two participants in the dementia group, with a diagnosis of dementia by a specialist, refused to complete the MoCA assessment; MoCA scores were only available for $5 \mathrm{PwD} .{ }^{\mathrm{b}} \mathrm{UAB}$-LSA was conducted twice with each participant, once at baseline, UAB-LSA (1), and once after 4 weeks of data collection, UAB-LSA (2). Two CTLs refused to complete the UAB-LSA (2); scores were only available for 6 CTLs.

period (i.e., global) or daily. The global indicators can reveal how participants' mobility patterns relate to their overall health, while the daily indicators can identify the magnitude of variability in both outdoor mobility and health outcomes over time [31]. Thus, a combination of daily and global indicators is considered in the proposed framework.

We propose a comprehensive set of GPS-based indicators that measure different dimensions of outdoor mobility as described above (shown in Table 2) $[39,40]$. The definitions of our proposed GPS-based indicators are presented in Table 3. To select these indicators, we searched the literature for articles that used GPS technology to measure older adults' outdoor mobility. We selected the indicators that were most frequently used in these articles (i.e., maximum distance, life space area, number of destinations, time spent at home, and time in vehicle). These common indicators were complemented with another 6 that we hypothesized are related to health outcomes in the dementia population (number of unique destinations, time doing out-of-home activities, time spent on foot, trip time period, outdoor activity duration timing, and activity types). For instance, the timing indicators (trip time period and activity duration timing) can be indicative of participation in day programs, and activity types can provide information about a person's current cognitive and physical abilities [41]. Finally, we included "radius of gyration," which is a common indicator used to measure typical distance travelled in human mobility research [40].

\section{Statistical Analysis}

To assess the differences in GPS-based indicators between PwD and cognitively intact CTLs, first, the Shapiro-Wilk test was performed to check the normality of the data. If the data were normally distributed in both groups, differences were examined using the Welch 2-sample $t$ test. Otherwise, the non-parametric MannWhitney test was performed. A $p$ value $<0.05$ was considered statistically significant between variables. For normally distributed indicators, to examine the between-group differences, we determined Hedges g, which is a corrected effect size estimate, rather than Cohen $d$, because it corrects biases due to small sample size. Hedge's g of $0.2-0.5$ is small, $0.5-0.8$ is moderate, and $>0.8$ is strong effect. For indicators that were not normally distributed, Cliff s Delta was chosen as a non-parametric estimate of effect size and calculated for each group [42]. Cliff's Delta values fall in the interval from -1 to +1 , where a Cliff s Delta of +1 or -1 indicates no overlap between the 2 groups, whereas a 0 Cliff's Delta indicates that group distributions overlap completely [43]. An absolute value of Cliff s Delta of $0.11-0.28$ is considered a small, $0.28-0.43$ is considered a medium, and $>0.43$ is considered a large effect.

\section{Results}

\section{Group Characteristics}

The participants completed 27-28 days of GPS data collection (PwD vs. CTL: $27.4 \pm 0.49$ vs. $27.8 \pm 0.43$ days). The characteristics of the individuals in each group are described in Table 4. The group differences in age ( $\mathrm{PwD}$ vs. CTL: $79.3 \pm 6.3$ vs. $72.9 \pm 5.9$ years) and education level (PwD vs. CTL: $14.1 \pm 3.4$ vs. $16.5 \pm 3.6$ years), although not statistically significant, were large. These differences may be due to the small sample size. PwD had a smaller proportion of females ( 43 vs. $50 \%$ females). The MoCA scores demonstrated cognitive impairment in PwD and normal cognitive function in the CTLs (MoCA scores: $19.4 \pm 2.4$ vs. $27.5 \pm 1.2$ ). PwD demonstrated a higher number of comorbidities compared to the CTLs (CCI: $2.14 \pm 1.6$ vs. $1.13 \pm 1.4$ ).

PwD displayed lower levels of instrumental ADL compared to the CTLs $(8.9 \pm 5.1$ vs. $14.0 \pm 0.0)$, but similar levels of ability to perform physical ADLs $(11.9 \pm 2.5$ vs. 


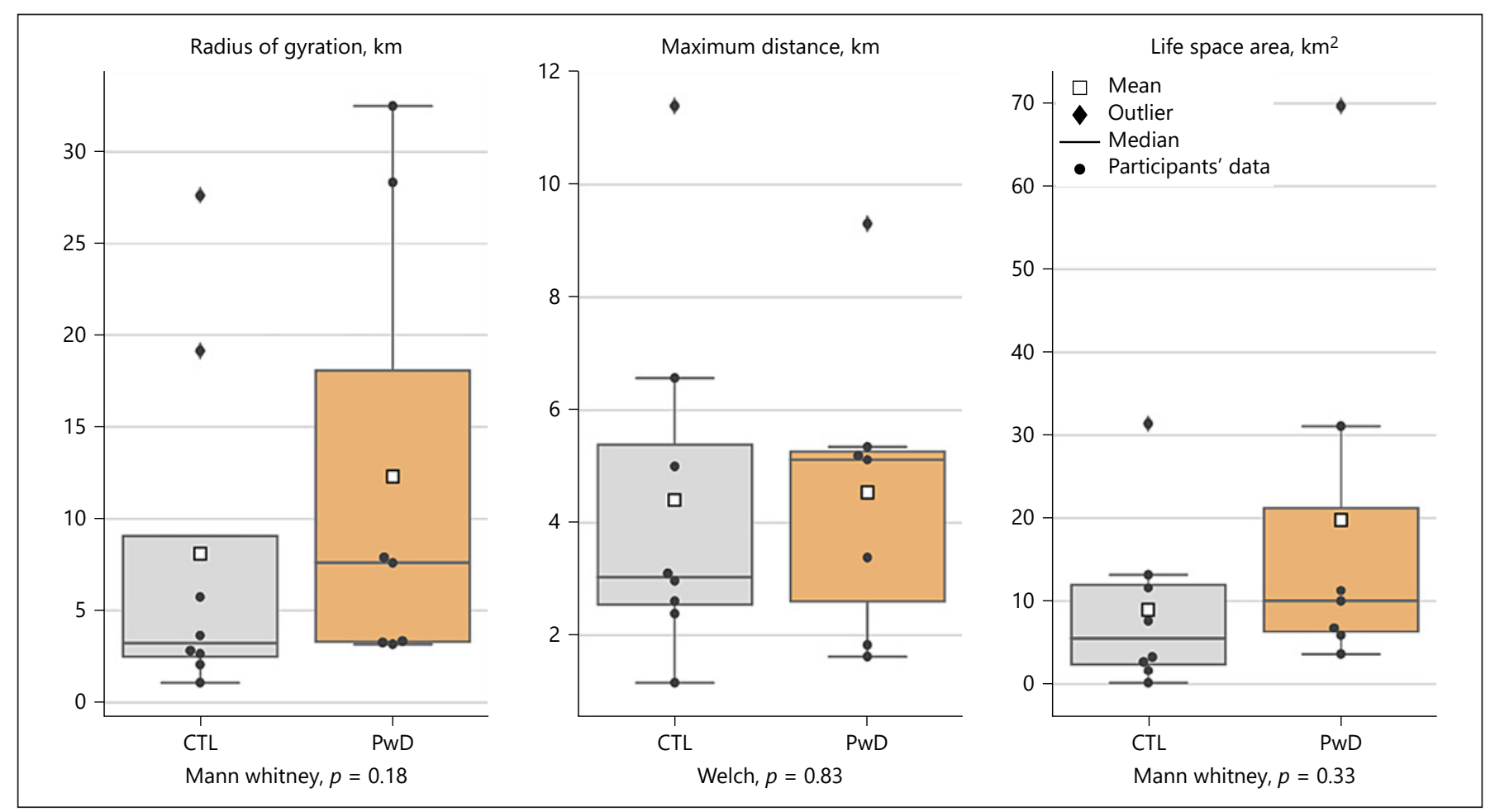

Fig. 1. Group comparison for the GPS measures describing the extent of outdoor mobility. GPS, global positioning system.

$13.8 \pm 0.7)$. PwD had lower levels of outdoor mobility compared to the CTLs, as measured by UAB-LSA conducted 2 times during the study period, but the differences were not statistically significant (UAB-LSA [1]: $54.9 \pm 28.7$ vs. $75.5 \pm 13.2$; UAB-LSA [2]: $52.5 \pm 21.0$ vs. $74.0 \pm 12.3$ ). Finally, there was no difference between the neighbourhood walk score of the 2 groups [38].

\section{Group Comparisons in Mobility}

Spatial Dimensions

Extent. Since the distributions of radius of gyration and life space area were not normal, the Mann-Whitney test was conducted, which indicated no statistically significant differences in the medians of radius of gyration (PwD vs. CTL: $\mathrm{Mdn}=7.60$ vs. $\mathrm{Mdn}=3.22, \mathrm{U}=16, p=$ $0.18)$ and daily life space area $(\mathrm{Mdn}=10.01 \mathrm{~km}$ vs. $\mathrm{Mdn}=5.46 \mathrm{~km}, p=0.46, \mathrm{U}=15, p=0.33$ ) between $\mathrm{PwD}$ and the CTLs. Analysis of between-group effects yielded an overall Cliffs Delta of -0.42 (95\% CI: -0.88 to 0.22 ) for radius of gyration and -0.32 (95\% CI: -0.86 to 0.33 ) for daily life space area. Furthermore, the maximum distance travelled was normally distributed according to the Shapiro-Wilk test, and the Welch 2-sample $t$ test indicated no statistically significant difference in the maxi- mum distance travelled (PwD vs. CTL: $4.54 \pm 2.62$ vs. 4.40 $\pm 3.28, t[13]=-0.23, p=0.83)$ between the 2 groups. The Hedge's g of -0.04 (95\% CI: -1.32 to 1 ) for maximum distance travelled indicates negligible effect size between the 2 groups. The group comparison results for these indicators are plotted in Figure 1.

Count. No count indicator significantly deviated from normality based on the Shapiro-Wilk test. The Welch 2 -sample $t$ test indicated no statistically significant differences in the total number of destinations (nDest: 100.29 \pm 58.86 vs. $124.5 \pm 34.87, t[10]=0.95, p=0.36)$ and the number of new destinations (nNewDest: $6.86 \pm 3.34$ vs. $10.38 \pm$ $4.98, t[13]=1.62, p=0.13)$ between groups. We obtained a small Hedge's g of 0.48 (95\% CI: -0.48 to 2.1$)$ and a moderate Hedge's $\mathrm{g} 0.77$ of (95\% CI: -0.12 to 2.1$)$ for in between group analyses for the total number of destinations and number of new destinations, respectively. The group comparison results for count indicators are plotted in Figure 2.

\section{Temporal Dimensions}

Duration. Shapiro-Wilk tests only identified non-normal distributions for the walking time indicator; the remaining duration indicators did not significantly deviate 


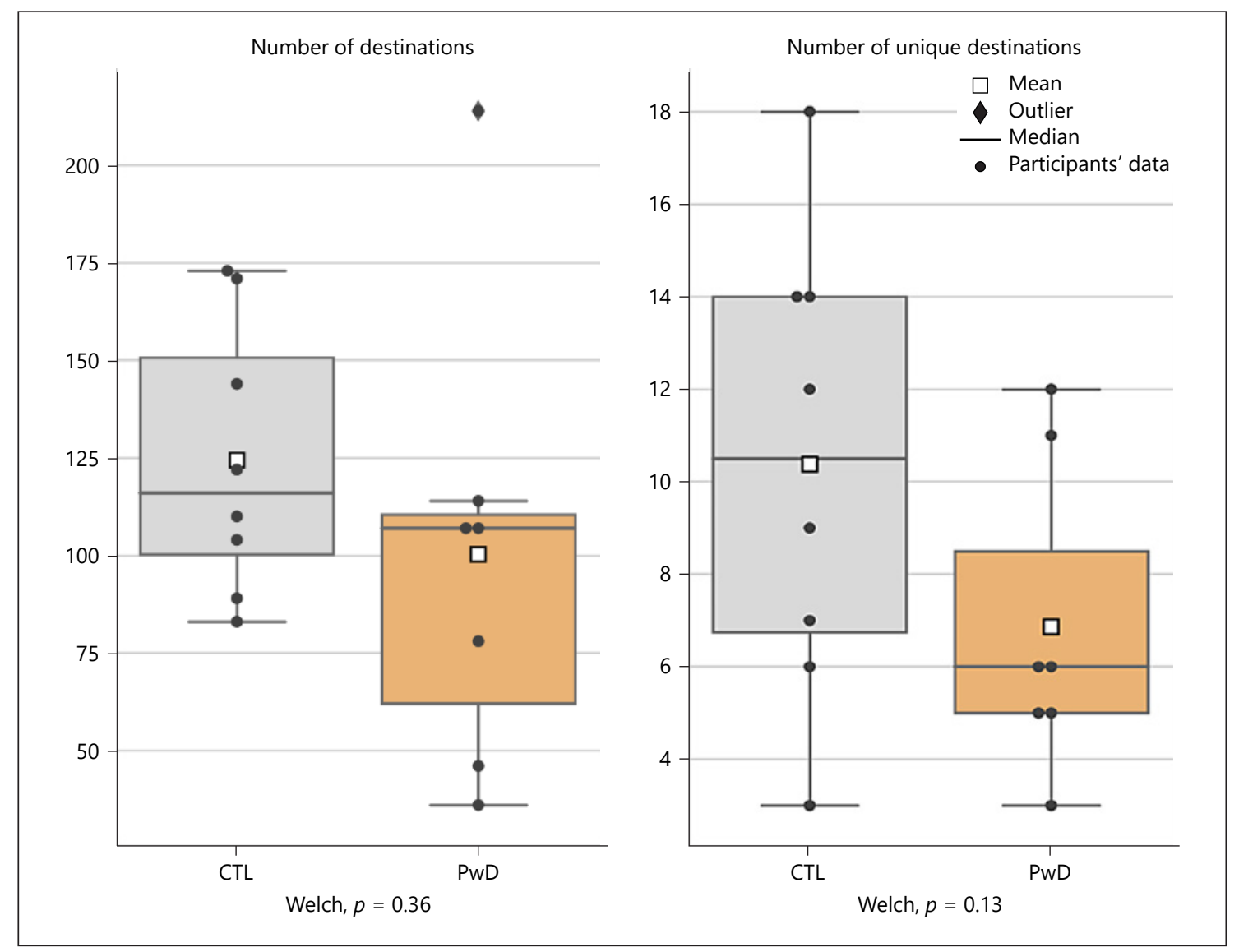

Fig. 2. Group comparison for the GPS measures describing the count of mobility-related events. GPS, global positioning system.

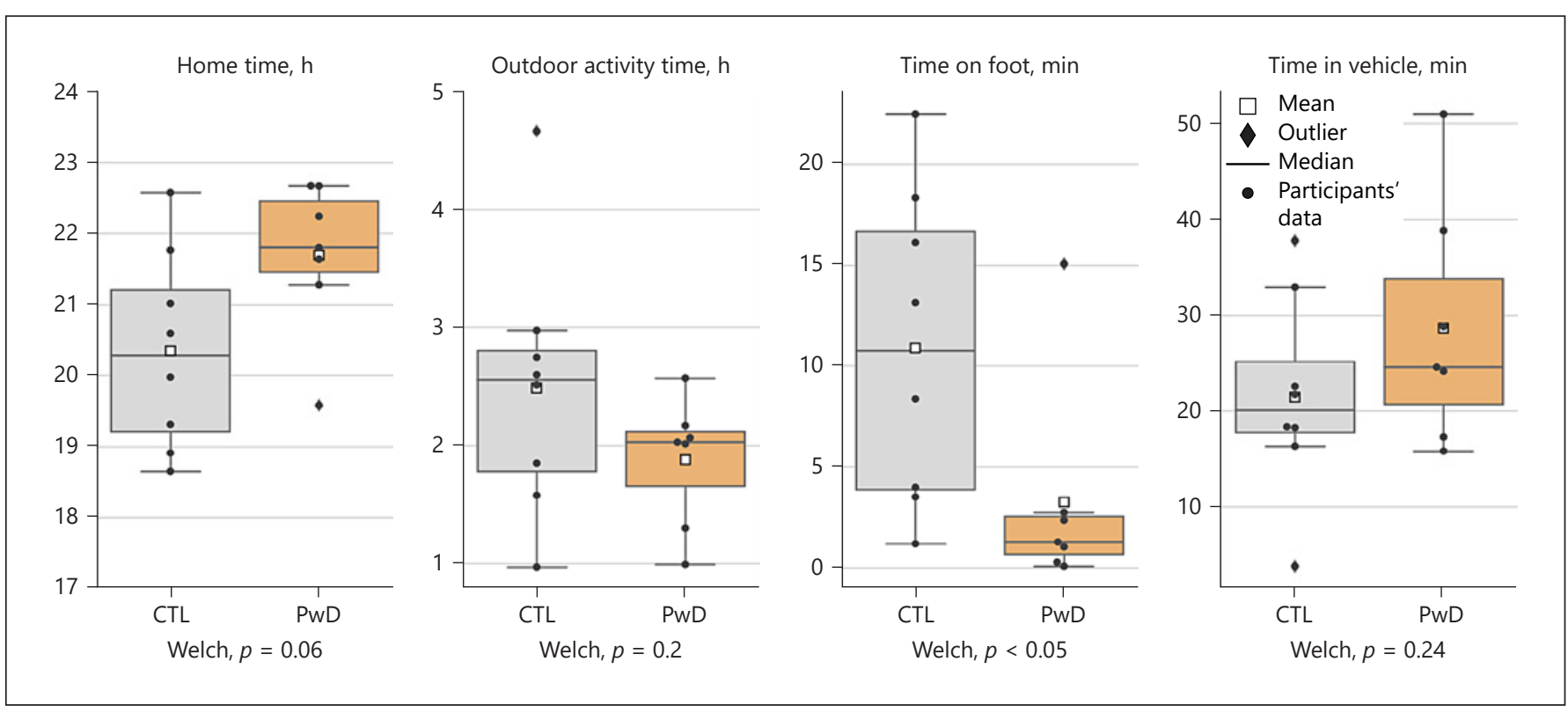

Fig. 3. Group comparison for the GPS measures describing the duration of mobility events. GPS, global positioning system.

Mobility Patterns of Older Adults with Dementia
Gerontology 2022;68:106-120

DOI: $10.1159 / 000515391$ 


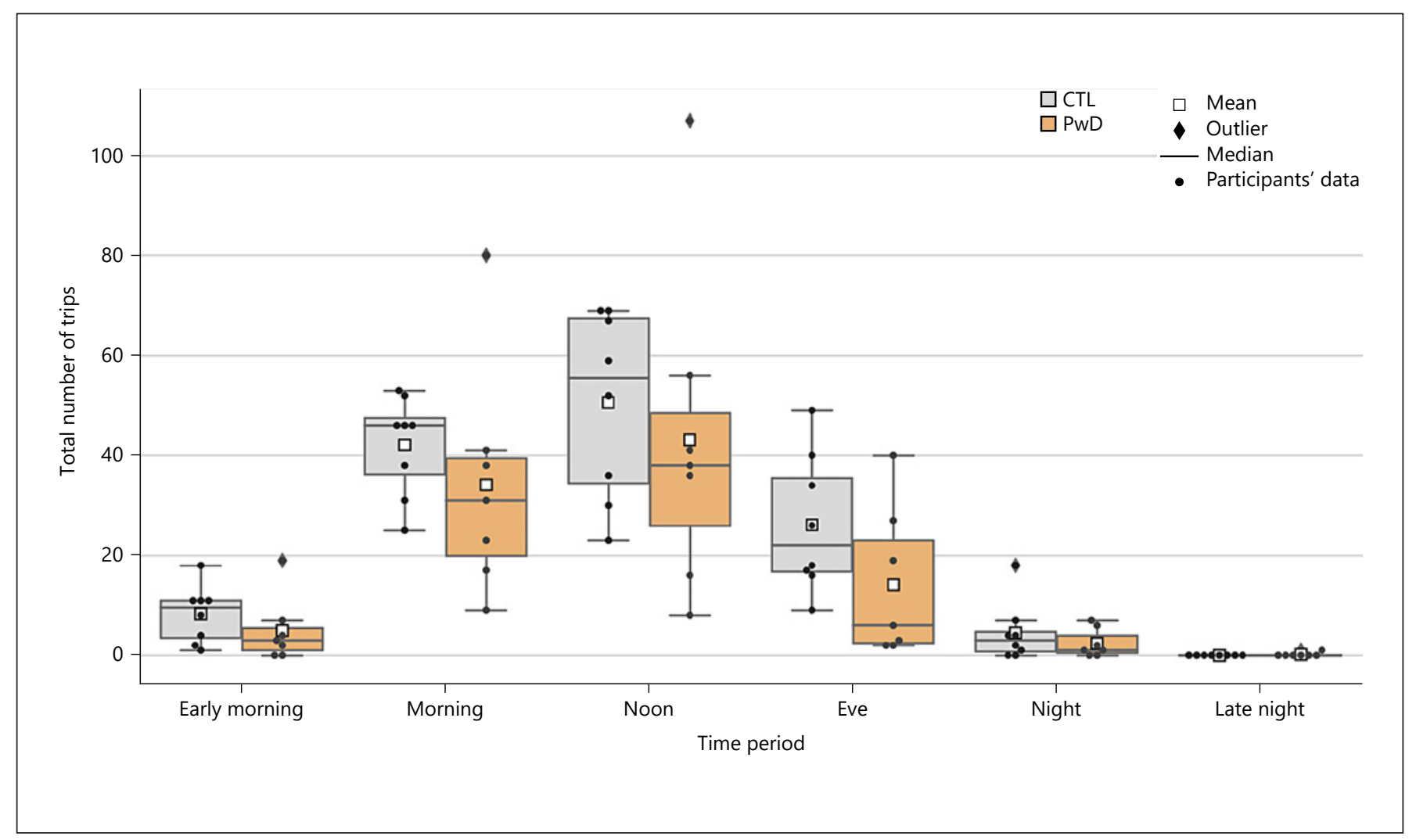

Fig. 4. Total number of trips by group in each time period.

from normality. Although PwD trended toward spending more time in home (Welch 2-sample $t$ test) $(1,301.92 \pm$ 64.10 vs. $1,221.08 \pm 83.71 \mathrm{~min}, t[13]=-2.11, p=0.06)$ and reduced time in outdoor activities compared to the CTL group $(112.64 \pm 32.48$ vs. $149.21 \pm 66.48 \mathrm{~min}$, $t[11]=1.38, p=0.2)$, these trends did not reach statistical significance. Analysis of between-group effects yielded an overall Hedge's g of -1.02 (95\% CI: -2.93 to -0.01 ) for time spent in home and 0.66 (95\% CI: -0.27 to 1.8 ) for time spent participating in outdoor activities.

The average daily walking time was significantly lower for PwD than for the CTL group (Mann-Whitney test) (PwD vs. CTL: $\mathrm{Mdn}=3.27$ vs. $\mathrm{Mdn}=10.80, \mathrm{U}=48, p=$ $0.02)$. Finally, no significant difference was exhibited in the average time spent in vehicles between the 2 groups (Welch 2 -sample $t$ test) (PwD vs. CTL: $28.64 \pm 12.45$ vs. $21.49 \pm 10.38 \mathrm{~min}, t[12]=-1.20, p=0.24)$. We obtained a large Cliff s Delta effect of 0.71 (95\% CI: 0.148-1) and a moderate Hedge's g effect of -0.59 (95\% CI: -1.62 to 0.48 )

\footnotetext{
${ }^{1}$ The findings remained unchanged when the suspected outliers in the figures were excluded from the analyses.
}

for average time spent walking and in vehicles, respectively. The group comparison results for these indicators are plotted in Figure $3 .^{1}$

Timing. Since only one late-night trip was recorded for both groups, this time period was not included in the between-group comparison analysis. Shapiro-Wilk tests identified non-normal distributions for early morning and night trips and normal distributions for the remaining timeframes. The "morning" and "noon" periods were when both PwD and the cognitively intact CTLs were most active. PwD showed a slight preference for trips that started at "noon." Although PwD displayed fewer early morning, morning, and evening trips compared to the CTL group, the differences were not statistically significant in the early morning group (Mann-Whitney test) (PwD vs. CTL: $\mathrm{Mdn}=3.0$ vs. $\mathrm{Mdn}=9.5, \mathrm{U}=48, p=0.02$ ), morning (Welch 2-sample $t$ test) (PwD vs. CTL: $34.1 \pm$ 23.2 vs. $42.1 \pm 10.0, t[8]=0.85, p=0.42)$, noon (Welch 2 -sample $t$ test) (PwD vs. CTL: $43.1 \pm 32.4$ vs. $50.6 \pm 18.6$, $t[9]=0.54, p=0.60)$, evening (Welch 2-sample $t$ test) (PwD vs. CTL: $14.14 \pm 15.0$ vs. $26.12 \pm 13.7, t[13]=1.60$, 


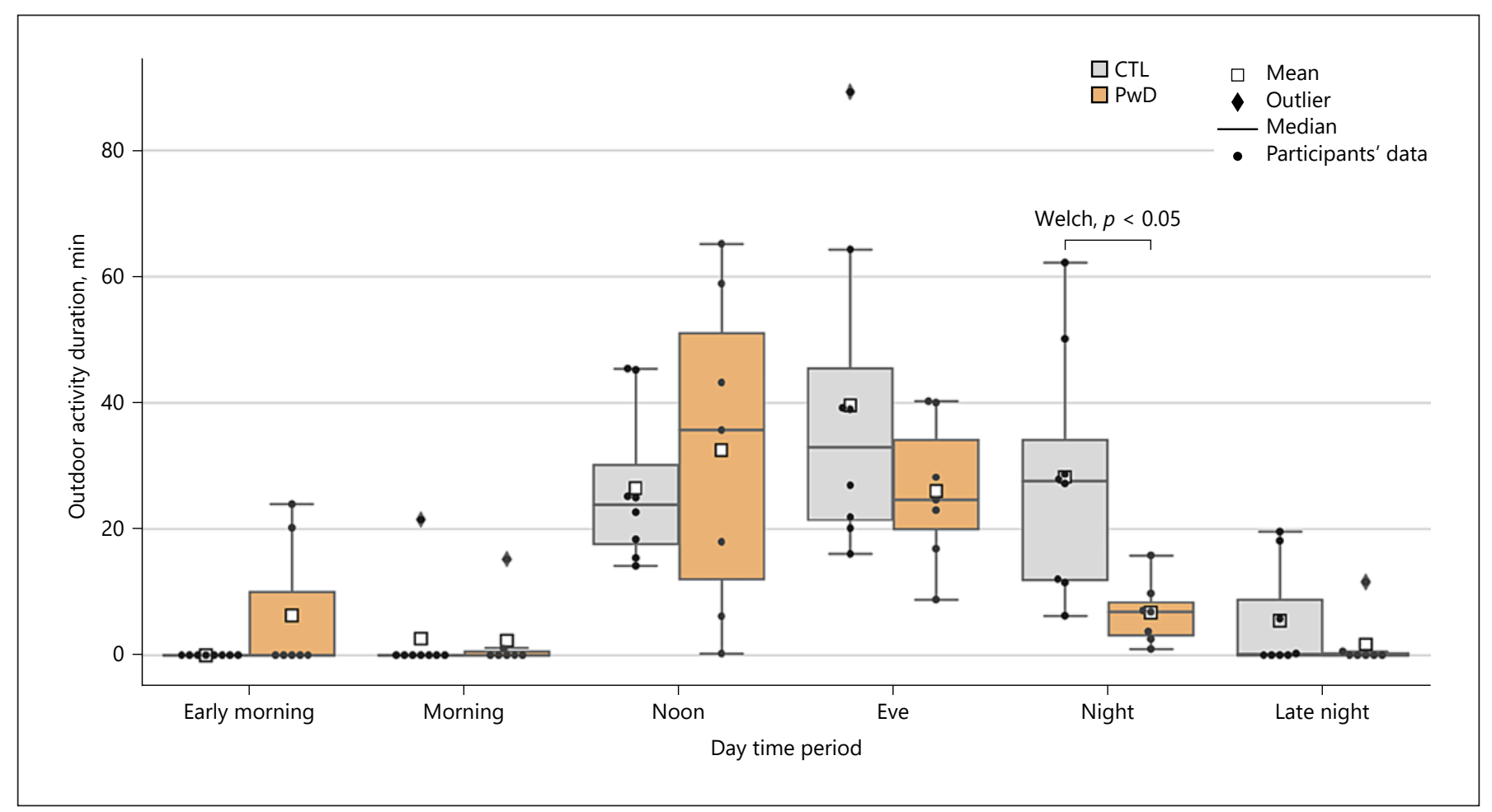

Fig. 5. Average activity duration by group in each time period.

$p=0.13$ ), and night (Mann-Whitney test) (PwD vs. CTL: $\mathrm{Mdn}=1.00$ vs. $\mathrm{Mdn}=3.00, \mathrm{U}=33, p=0.60)$. Furthermore, effect size analyses yielded a Cliff's Delta effect of 0.39 ( $95 \%$ CI: -0.24 to 0.911 ) for early morning trips, and Hedge's g effects of 0.42 (95\% CI: -0.61 to 2.88 ), 0.27 ( $95 \%$ CI: -0.71 to 1.85 ), and 0.79 (95\% CI: -0.17 to 2.55 ) for morning, noon, and evening trips. We also obtained a Cliff's Delta effect size of 0.18 (95\% CI: -0.48 to 2.42 ) for night trips. The group comparison results for the active time period indicator are provided in Figure 4.

Since only a few early morning, morning, and latenight outdoor activities were recorded, these time periods were not considered for between-group comparisons. Shapiro-Wilk tests identified normal distributions for noon, evening, and night periods. The duration of the outdoor activities starting at "noon" was highest for $\mathrm{PwD}$, while the duration of outdoor activities starting in the "evening" was highest for the CTL group. PwD demonstrated reduced activity duration for activities that were initiated at "night" compared to the CTLs (Welch 2-sample $t$ test) (PwD vs. CTL: $6.72 \pm 5.03$ vs. $28.27 .1 \pm 19.51, t[9]=3.02, p<0.05)$, with a large Hedge's g effect of 1.42 (95\% CI: 0.85-1.09). However, the differences for "noon" or "evening" timeframes were not statistically significant (Welch 2 -sample $t$ test) (PwD vs. CTL: noon: $32.50 \pm 25.28$ vs. $26.4 \pm 12.35$, $t[8]=-0.57, p=0.58$; evening: $26.0 \pm 11.48$ vs. $39.61 \pm$ $25.32, t[11]=1.37, p=0.21)$. Analysis of betweengroup effects yielded a small Hedge's g of -0.29 (95\% CI: -1.85 to 0.92 ) and a moderate Hedge's g of 0.65 ( $95 \%$ CI: -0.29 to 1.66 ) for average activity durations in "noon" and "evening," respectively. The group comparison results for outdoor activity duration by the time period are provided in Figure 5.

\section{Semantic Dimensions}

The group comparison results for activity types are plotted in Figure 6. Shapiro-Wilk tests identified nonnormal distributions for medical, religious, and sport activities and normal distributions for the remaining activity types. PwD attended a higher proportion of medicaland health-related activities (Mann-Whitney test) (PwD vs. CTL: $\mathrm{Mdn}=0.23$ vs. $\mathrm{Mdn}=0.11, \mathrm{U}=6, p=0.01$ ) with strong a Cliff s d effect of 0.71 (95\% CI: 0.34-1) and a lower proportion of sport activities (Mann-Whitney test) (PwD vs. CTL: $\mathrm{Mdn}=0.0$ vs. $\mathrm{Mdn}=0.08, \mathrm{U}=48, p=0.01$ ) with a strong Cliff s d effect of -0.78 ( $95 \% \mathrm{CI}:-1$ to -0.32$)$ compared to the CTL group. The differences between re- 


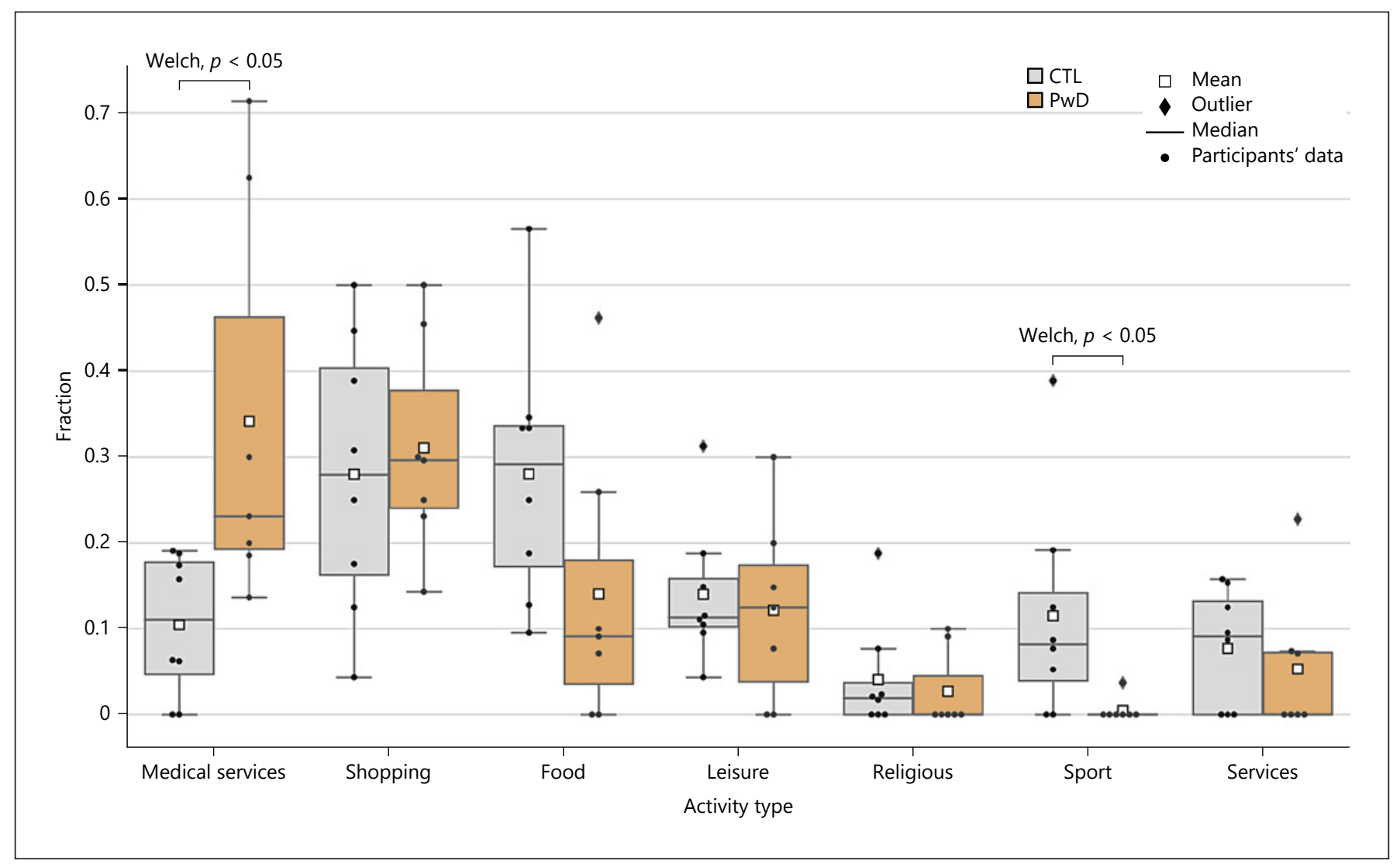

Fig. 6. Comparison results for activity types.

maining activity types were not statistically significant. Furthermore, effect size analyses yielded a large Hedge's g effect of 0.83 (95\% CI: -0.09 to 2.72 ) for food activities and negligible to small effects for the remaining activity types.

\section{Discussion}

This study presents a framework for comparing outdoor mobility patterns of cognitively intact older adults and older adults with dementia based on a comprehensive set of GPS-based indicators. To provide an objective and inclusive view of the outdoor mobility of older adults with dementia, we focused on mobility indicators derivable from GPS data and domain knowledge.

Our proposed framework extends the available literature on life space patterns of older adults with dementia by introducing GPS-based indicators that measure various dimensions of mobility that may be impacted by cognitive decline. Although previous studies have shown that greater complexity of living environments can protect against cognitive decline [22], no study, to date, has attempted to capture this complexity. Tung et al. [24] proposed indicators to measure the extent of movements in the environment of older adults with Alzheimer's disease using GPS. Our framework builds on previous findings by introducing GPS-based indicators that measure more in-depth characteristics of mobility. For instance, in addition to spatial dimensions, our framework examines temporal and semantic dimensions of outdoor mobility and provides evidence for associations between all aspects of outdoor mobility and cognitive function. It is, in fact, important to consider these different dimensions simultaneously in order to gain a comprehensive understanding of outdoor mobility behaviours of older adults with dementia. For instance, although previous studies show that the larger extent of outdoor mobility (e.g., life space area) could be indicative of improved cognition [24], in our small sample, no statistically significant differences were observed in indicators measuring the spatial dimensions of outdoor mobility. This, however, does not imply similar levels of outdoor mobility between 
PwD and cognitively intact CTLs. In fact, the application of our proposed framework demonstrates several other significant differences. For instance, our results suggest that longer duration of outdoor walking time is associated with intact cognition $(p<0.05)$ with large effect (Cliff s delta $=-0.71)$, meaning there is a $71 \%$ chance that an individual with dementia has a lower daily walking time than a cognitively intact individual. This may be at least partly due to spatial disorientation, which is a common symptom of dementia influencing outdoor wayfinding performance of individuals and increasing their risk of getting lost [10]. Furthermore, our sample of PwD also demonstrated a slight trend toward spending more time at home $(p<0.10)$ compared to the CTLs with a large effect (Hedge's $g=-1.02$ ); thus, someone from the dementia group is more likely to spend more time at home than $84 \%$ of the cognitively intact CTLs. This finding, consistent with the available literature [10], highlights the need to support $\mathrm{PwD}$ such that they can continue living at home but not be confined to it. Looking further into the timing indicators, we observed that the 2 individuals in the dementia group, who participated in regular adult day programs, also exhibited the highest duration of activities in the timeframe from 12 to 4 p.m., which also matched the timing of their programs. Our results also suggest that longer outdoor activities at night (i.e., after 8 p.m.) are associated with individuals with intact cognition $(p<0.05)$ and that $\mathrm{PwD}$ tend to participate in more medical-related activities $(p<0.05)$ and fewer sports activities compared to the cognitively intact CTLs $(p<0.05)$. Overall, we hypothesize that with a larger sample, these differences between mobility behaviours would become more apparent.

Another novelty of our framework is the explicit and detailed categorization of semantic aspects related to an individual's motivations and intentions such as activity types and transportation modes. Most previous classification frameworks for mobility indicators do not characterize older adults' outdoor mobility according to its semantic aspects [30]. The few studies that investigate semantic indicators consider only a few indicators and categories [28]. For example, they only distinguish between physically demanding activities and cognitively demanding activities [28]. These studies also require the participants to recall and report their activity types and are thus subject to recall bias among cognitively impaired populations $[28,29]$. Considering activity types is of particular importance in the context of cognitive impairment because older adults' specific types of destinations and activities are influenced by their cognitive status [44]. For instance, in

Mobility Patterns of Older Adults with Dementia our sample, the $2 \mathrm{PwD}$ with the largest life space areas (LSA: 69.9 and $31.1 \mathrm{~km}^{2}$ ) visited the fewest number of destinations (nDest: 36 and 46). However, their furthest destinations leading to larger life space areas were to medical locations outside their immediate environments. Therefore, one can presume that, for $\mathrm{PwD}$, a smaller life space in a dense age-friendly environment with more diverse activities can be advantageous. To make this distinction, it is important to transition from traditional life space constructs that only consider the extent of outdoor movements to constructs that capture more complex dimensions of outdoor mobility to be able to promote PwD's engagement in trips and activities that improve their health and social connection. Overall, looking at the contextual information inferred from GPS trajectories, our results suggest that $\mathrm{PwD}$ participated in significantly more medical- and health-related activities $(p<0.05)$ and fewer sport activities $(p<0.05)$ compared to cognitively intact CTLs. The observed effect of -0.78 in medical-related activities may be at least partly due to participation of $\mathrm{PwD}$ in day programs that are often located in health centres. Another semantic indicator included in our framework is the transportation mode. With respect to transportation modes, we distinguished between walking and passive modes (i.e., trips in vehicles). This distinction is important because walking is a major contributor of physical activity among older adults and may help maintain cognitive function [45]. To the best of our knowledge, the detailed and fully autonomous categorization of semantic dimensions from GPS data as a part of the proposed classification framework has not been represented in previous mobility frameworks presented in the gerontology literature.

Our proposed GPS-based framework offers new perspectives on the development of assistive technology devices that support the unmet outdoor mobility needs of people living with dementia. By characterizing and learning mobility patterns of $\mathrm{PwD}$, we can explore the possibility of using machine-learning algorithms to create personalized models that predict the whereabouts of PwD and detect movements that may correspond to geographic disorientation. These personalized predictive models can support PwD to safely and freely navigate in the outdoor environment and locate them in case they go missing; thus, providing both the person with dementia and their caregiver more confidence in using the outdoor environment independently. However, despite all the advantages and promises of tracking devices for PwD and their caregivers, 3 key limitations should be considered before their use. First, the 
power of these devices in providing continuous and remote monitoring of $\mathrm{PwD}$ has privacy implications for the older adult and can lead to potential information leakage about users' whereabouts. Second, GPS signals are less accurate in some areas and unavailable in indoor environments because of signal interference and obstruction. Third, PwD may lose, remove, or forget to charge these tracking devices.

Finally, the findings of the current study need to be considered in light of a number of limitations, which can be addressed in future research. First, the small sample in this study, though effective for establishing the feasibility of the presented framework, lacks generalizability. Future studies should seek to achieve statistical power to allow for generalizable findings. The results obtained in this study could be skewed because of the unmatched age in dementia and control groups. Future studies with a larger sample should investigate the role of age and gender in the travel patterns of the 2 groups, since gender and age may contribute to various factors, including social and cultural norms regarding daily mobility behaviour. Second, no corrections were made for multiple comparisons between the 2 groups. Thus, further studies on larger sample sizes are required to provide a comprehensive analysis of outdoor mobility behaviours of older adults with dementia. Third, since individuals at different stages of dementia have different mobility characteristics, future studies should consider investigating mobility profiles of individuals with mild, moderate, and severe dementia separately. Fourth, our proposed set of indicators was limited to mobility patterns that were derivable from GPS data and domain knowledge. Therefore, additional semantic aspects related to others who accompanied individuals on their trips were not included. The inclusion of social network measures in the outdoor mobility construct could further extend the current framework. This could be done by combing various sensors including audio data or accelerometers, or by incorporating location data from family members. For older adults with dementia who have lost their driving privileges, data about with whom they are travelling can yield a better understanding of their access to the available transportation and dependence on their caregivers.

\section{Conclusion}

The present study suggests that to overcome the apparent limitations of traditional life space measures and to objectively assess mobility behaviours of cognitively impaired populations, GPS technology can be used. This study presents a framework that allows a thorough comparison of outdoor mobility patterns of older adults with dementia and cognitively intact older adults using a set of indicators reflecting different dimensions of outdoor mobility. The proposed framework finds that in addition to determining the extent of the movements in the environment, which has been a primary focus of articles on older adult GPS-based life space assessments, GPS tracking devices can be used to automatically infer more in-depth information about the user's mobility behaviour. This information ranges from semantic characteristics such as the user's activity types to temporal characteristics indicating the timing of the user's out-of-home trips. By moving from life space constructs that focus on the extent of mobility to multidimensional outdoor mobility constructs, we can better promote active lifestyles and social connections among the population of older adults with dementia. Furthermore, the proposed framework demonstrates the information that can be derived from raw trajectory data, and its application to a larger sample can help discriminate against aspects of mobility that are more relevant to cognitive health. Future work will focus on the development of predictive machine-learning mobility models that learn mobility habits of $\mathrm{PwD}$ from the proposed framework, using a larger sample that achieves statistical power, and then infer their future destinations and intentions. By predicting future mobility patterns, we can identify when a user diverges from a predefined destination and assist them appropriately, if required; thus, we can move toward intelligent navigation assistance systems that support outdoor mobility needs of PwD to improve safety and autonomy.

\section{Acknowledgement}

We would like to thank all the participants and their families who kindly took the time to participate in the study. We would like to express our gratitude to the SafeTracks GPS Canada Inc. for its technical support. We would also like to acknowledge Ms. Bing Ye, who contributed to the REB approval process and data collection.

\section{Statement of Ethics}

The present study was approved by the Ethics Committee of the Baycrest Hospital and the University Health Network (REB \#1538). Written informed consent was obtained from all the participants.
Bayat/Naglie/Rapoport/Stasiulis/ Widener/Mihailidis 


\section{Conflict of Interest Statement}

The authors have no conflicts of interest to declare.

\section{Funding Sources}

This project is part of the Canadian Consortium on Neurodegeneration in Aging (CCNA; Grant No. 201312CNA-322265CNA-CFAF-32054). The CCNA is supported by a grant from the Canadian Institute of Health Research (CIHR) with funding from several partners. This research was also supported with funding from the AGE-WELL NCE. Gary Naglie was supported by the George, Margaret and Gary Hunt Family Chair in Geriatric Medicine, University of Toronto.

\section{Author Contributions}

S.B., G.N., M.J.R., E.S., and A.M. designed the study. S.B., M.W., and A.M. contributed to the design of the framework. All authors contributed to the interpretation of the results. S.B. wrote the manuscript with input from all authors. All authors provided critical feedback and helped shape the research, analysis, and manuscript.

\section{References}

1 Xiang X, Cheng J. Trajectories of major depression in middle-aged and older adults: a population-based study. Int J Geriatr Psychiatry. 2019;34(10):1506-14.

2 Xiang X, An R, Oh H. The bidirectional relationship between depressive symptoms and homebound status among older adults. J Gerontol B Psychol Sci Soc Sci. 2020 Jan 14 75(2):357-66.

3 Welch V, Howe TE, Marcus S, Mathew CM, Sadana R, Rogers M, et al. PROTOCOL: health, social care and technological interventions to improve functional ability of older adults: evidence and gap map. Campbell Syst Rev. 2019;15(4):e1054.

4 Tivadar BK. Physical activity improves cognition: possible explanations. Biogerontology. 2017;18(4):477-83.

5 Zhao E, Tranovich MJ, Wright VJ. The role of mobility as a protective factor of cognitive functioning in aging adults: a review. Sports Health. 2014 Jan;6(1):63-9.

6 Oswald F, Wahl H-W, Voss E, Schilling O, Frey$\operatorname{tag} \mathrm{T}$, Auslander G, et al. The use of tracking technologies for the analysis of outdoor mobility in the face of dementia: first steps into a project and some illustrative findings from Germany. J Hous Elderly. 2010 Feb;24(1):55-73.

7 Spatial navigation deficits: overlooked cognitive marker for preclinical Alzheimer disease? | Nature Reviews Neurology [Internet]. [cited 2020 Aug 12]. Available from: https://www. nature.com/articles/s41582-018-0031-x.

8 Babulal GM, Stout SH, Benzinger TLS, Ott BR, Carr DB, Webb M, et al. A naturalistic study of driving behavior in older adults and preclinical Alzheimer disease: a Pilot Study. J Appl Gerontol. 2019 Feb;38(2):277-89.

9 Cerman J, Andel R, Laczo J, Vyhnalek M, Nedelska Z, Mokrisova I, et al. Subjective spatial navigation complaints: a frequent symptom reported by patients with subjective cognitive decline, mild cognitive impairment and Alzheimer's disease. Curr Alzheimer Res. 2018 Mar;15(3):219-28
10 Duggan S, Blackman T, Martyr A, Schaik PV. The impact of early dementia on outdoor life: a "shrinking world"? Dementia and geriatric cognitive disorders. 2008 May;7(2):191-204.

11 Meuser TM, Carr DB, Berg-Weger M, Niewoehner P, Morris JC. Driving and dementia in older adults: implementation and evaluation of a continuing education project. Gerontologist. 2006 Oct;46(5):680-7.

12 EnviroStats: the use of transportation by seniors in Canada [Internet]. [cited 2020 Aug 12]. Available from: https://www150.statcan. gc.ca/n1/pub/16-002-x/2010004/part-partie3-eng.htm.

13 Bailey LA. Aging Americans: stranded without options. 2004.

14 Stalvey BT, Owsley C, Sloane ME, Ball K. The life space questionnaire: a measure of the extent of mobility of older adults. J Appl Gerontol. 1999; 18:460-78.

15 Tsai LT, Portegijs E, Rantakokko M, Viljanen A, Saajanaho M, Eronen J, et al. The association between objectively measured physical activity and life-space mobility among older people. Scand J Med Sci Sports. 2015 Aug; 25(4):e368-73.

16 Polku H, Mikkola TM, Portegijs E, Rantakokko M, Kokko K, Kauppinen M, et al. Life-space mobility and dimensions of depressive symptoms among community-dwelling older adults. Aging Ment Health. 2015 Sep;19(9):781-9.

17 Makhija SK, Gilbert GH, Clay OJ, Matthews JC, Sawyer P, Allman RM. Oral health-related quality of life and life-space mobility in community-dwelling older adults. J Am Geriatr Soc. 2011 Mar;59(3):512-8.

18 Auais M, Alvarado B, Guerra R, Curcio C, Freeman EE, Ylli A, et al. Fear of falling and its association with life-space mobility of older adults: a cross-sectional analysis using data from five international sites. Age Ageing. 2017 May;46(3):459-65

19 Crowe M, Andel R, Wadley VG, Okonkwo OC, Sawyer P, Allman RM. Life-space and cognitive decline in a community-based sam- ple of African American and caucasian older adults. J Gerontol A Biol Sci Med Sci. 2008 Nov;63(11):1241-5.

20 May D, Nayak US, Isaacs B. The life-space diary: a measure of mobility in old people at home. Int Rehabil Med. 1985;7(4):182-6.

21 Zenk SN, Matthews SA, Kraft AN, Jones KK How many days of global positioning system (GPS) monitoring do you need to measure activity space environments in health research? Health Place. 2018 May;51:52-60.

22 Allman RM, Sawyer P, Roseman JM. The UAB Study of Aging: background and insights into life-space mobility among older Americans in rural and urban settings. Aging Health. 2006 May;2(3):417-29.

23 Shoval N, Wahl H-W, Auslander G, Isaacson M, Oswald F, Edry T, et al. Use of the global positioning system to measure the out-ofhome mobility of older adults with differing cognitive functioning. Ageing Soc. $2011 \mathrm{Jul}$; 31(5):849-69.

24 Tung JY, Rose RV, Gammada E, Lam I, Roy EA, Black SE, et al. Measuring life space in older adults with mild-to-moderate Alzheimer's disease using mobile phone GPS. Gerontology. 2014;60(2):154-62.

25 Phillips ML, Hall TA, Esmen NA, Lynch R, Johnson DL. Use of global positioning system technology to track subject's location during environmental exposure sampling. J Expo Anal Environ Epidemiol. 2001 Jul;11(3):20715.

26 Schenk AK, Witbrodt BC, Hoarty CA, Carlson RH, Goulding EH, Potter JF, et al. Cellular telephones measure activity and lifespace in community-dwelling adults: proof of principle. J Am Geriatr Soc. 2011 Feb;59(2):345-52.

27 Boissy P, Brière S, Hamel M, Jog M, Speechley $\mathrm{M}$, Karelis A, et al. Wireless inertial measurement unit with GPS (WIMU-GPS): wearable monitoring platform for ecological assessment of lifespace and mobility in aging and disease. Conf Proc IEEE Eng Med Biol Soc. 2011;2011:5815-9. 
28 Wettstein M, Wahl HW, Diehl MK. A multidimensional view of out-of-home behaviors in cognitively unimpaired older adults: examining differential effects of socio-demographic, cognitive, and health-related predictors. Eur J Ageing. 2014 Jun;11(2):141-53.

29 Perchoux C, Kestens Y, Thomas F, Van Hulst A, Thierry B, Chaix B. Assessing patterns of spatial behavior in health studies: their sociodemographic determinants and associations with transportation modes (the RECORD Cohort Study). Soc Sci Med. 2014 Oct;119: 64-73.

30 Brusilovskiy E, Klein LA, Salzer MS. Using global positioning systems to study healthrelated mobility and participation. Soc Sci Med. 2016;161:134-42.

31 Fillekes MP, Giannouli E, Kim EK, Zijlstra W, Weibel R. Towards a comprehensive set of GPS-based indicators reflecting the multidimensional nature of daily mobility for applications in health and aging research. Int $J$ Health Geogr. 2019 24;18(1):17.

32 Wettstein M, Wahl HW, Shoval N, Oswald F, Voss E, Seidl U, et al. Out-of-home behavior and cognitive impairment in older adults: findings of the SenTra Project. J Appl Gerontol. 2015 Feb;34(1):3-25.

33 Nielsen J. How many test users in a usability study? [Internet]. Nielsen Norman Group. [cited 2020 Jan 28]. Available from: https:// www.nngroup.com/articles/how-many-testusers/.
34 Nasreddine ZS, Phillips NA, Bédirian V, Charbonneau S, Whitehead V, Collin I, et al. The Montreal Cognitive Assessment, MoCA: a brief screening tool for mild cognitive impairment. J Am Geriatr Soc. 2005 Apr;53(4): 695-9.

35 Fillenbaum GG. Multidimensional functional assessment of older adults: the Duke Older Americans Resources and Services procedures. Hillsdale: Erlbaum; 1988. [cited 2020 Aug 18]. Available from: https://scholar. google.com/scholar_lookup?title=Multidime nsional+Functional+Assessment+of+Older+ Adults: +The+Duke+Older+Americans+Res ources + and +Services + Procedures \& author $=\mathrm{G}+$ Fillenbaum \& publication year $=1988 \&$

36 Crook TH, Feher EP, Larrabee GJ. Assessment of memory complaint in age-associated memory impairment: the MAC-Q. Int Psychogeriatr. 1992 Sep;4(2):165-76.

37 Quan H, Li B, Couris CM, Fushimi K, Graham $\mathrm{P}$, Hider $\mathrm{P}$, et al. Updating and validating the charlson comorbidity index and score for risk adjustment in hospital discharge abstracts using data from 6 countries. Am J Epidemiol. 2011 Mar;173(6):676-82.

38 Walk Score Methodology [Internet]. Walk Score. [cited 2020 Jan 28]. Available from: https: //www.walkscore.com/methodology. shtml.
39 Bayat S, Naglie G, Rapoport MJ, Stasiulis E, Chikhaoui B, Mihailidis A. Inferring destinations and activity types of older adults from GPS data: algorithm development and validation. JMIR Aging. 2020;3(2):e18008.

40 Pappalardo L, Simini F. Data-driven generation of spatio-temporal routines in human mobility. Data Min Knowl Disc. 2018 May; 32(3):787-829.

41 Buettner LL, Fitzsimmons S. Activity calendars for older adults with dementia: what you see is not what you get. Am J Alzheimers Dis Other Demen. 2003 Jul;18(4):215-26.

42 Cliff N. Dominance statistics: ordinal analyses to answer ordinal questions. Psychological Bulletin. 1993;114(3):494-509.

43 Macbeth G, Razumiejczyk E, Ledesma RD. Cliff's Delta Calculator: a non-parametric effect size program for two groups of observations. Universitas Psychologica. 2011 May; 10(2):545-55.

44 Chudyk AM, Winters M, Moniruzzaman M, Ashe MC, Gould JS, McKay H. Destinations matter: the association between where older adults live and their travel behavior. J Transp Health. 2015 Mar;2(1):50-7.

45 Weuve J, Kang JH, Manson JE, Breteler MM, Ware JH, Grodstein F. Physical activity, including walking, and cognitive function in older women. JAMA. 2004 Sep;292(12): 1454-61. 